\title{
Ensemble Learning Techniques for Appendicitis Prediction
}

\author{
K. Sathiyakumarir \\ Assistant Professor \\ P S G R Krishnammal \\ College for Women
}

\begin{abstract}
Appendicitis leftovers the most common cause of decrease belly ache. It retains a common look at all ages. The appendix is an attachment or adjunct like shape. it's miles a wormlike stomach diverticulum extending from the blind cease of the cecum; it varies in period and leads to a blin extremity. Early and correct analysis of appendicitis can lower the contamination and clinic cost by using lowering the put off in prognosis of appendicitis and its related headaches. accurate prognosis of appendicitis is a tough trouble in exercise especially if the patient is just too young or pregnant girls in that radiological check have excessive risk. thus, ultrasonography image evaluation is a good way to reduce the problem. This work affords an attempt to diagnose the appendicitis with the aid of extracting appendix of different levels from the stomach ultrasound picture. Diverse filtering techniques like LEE and FROST strategies are used for noise removal and Marker-controlled Watershed method is used for segmentation of appendicitis. The vicinity of interest (ROI) approach is used to extract the accurate portion of appendix photograph. The feel functions of the segmented ROI are Gary degree Co-prevalence Matrix (GLCM) and form capabilities are extracted for the future cause of classifying appendicitis. In the end, the ensemble gaining knowledge of set of rules is used to classify appendicitis appropriately via the use of an AdaBoost technique. The AdaBoost technique is evaluated the usage of various measures like Resubstitution Loss mistakes, Generalization mistakes, cross-Validation errors, and schooling errors. It gives very low loss errors rate.
\end{abstract}

\section{Keywords}

Appendicitis Prediction

\section{INTRODUCTION}

The two maximum common imaging tests used are the ultrasound and laptop tomography (CT test). CT experiment has been proven to be more correct than ultrasound in detecting acute appendicitis. But, ultrasound may be favored because the first imaging test in children and pregnant ladies because of the risks related to radiation exposure from CT scans the appendix is an appendage or appendix like shape. It's miles a wormlike intestinal diverticulum extending from the blind gives up of the cecum; it varies in the period and ends in a blind extremity [1]. The appendix is a part of the cecum from which it originates where the three tenia coli coalesce at the distal issue of the cecum. Not relatively, the appendix resembles the cecum histologically and consists of round and longitudinal muscle layers. The appendix arises from the cecum about $2.5 \mathrm{~cm}$ below the ileocecal valve. It varies in the period from entire agenesis to greater than $30 \mathrm{~cm}$, however, it is also 5 to $10 \mathrm{~cm}$ in duration. The imply width is 0. five to one. $0 \mathrm{~cm}[2]$. The primary thrust of activities main to the development of acute appendicitis lies in the appendix developing a compromised blood supply because of obstruction of its lumen and will become very at risk of invasion by using micro organism located within the gut usually. Obstruction of the appendix lumen by way of faecolith, enlarged lymph node, worms, tumour, regular mucus secretions preserve within the lumen of the appendix or indeed overseas gadgets, brings about a raised intra-luminal strain, which causes the wall of the appendix to grow to be distended, as a consequence causing further buildup of intraluminal pressures [3]. Through the usage of ultrasound to diagnose appendicitis, visualizing still remains a problem. A regular appendix is tough to be detected in ultrasound photograph so any visualize appendix in ultrasound picture is taken into consideration as ordinary [4]. The identical assessment is authentic for the patient with obese.

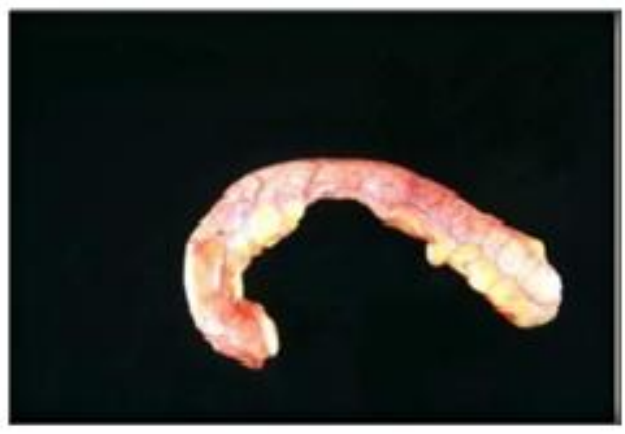

(a)

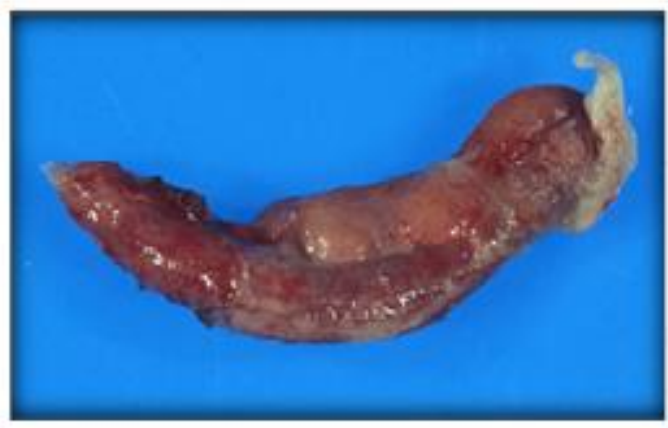

(b)

Fig1. (a) Normal appendix (b) Appendix inflammation

\section{Statistical analysis}

A total of 2402 appendectomy operations has been located since the last five years (2006-2010). among them, 1114 cases were males $(46 \%)$ and 1288 have been ladies (54\%).total variety of $1961(81.6 \%)$ instances of appendectomies have been completed for the removal of an infected appendix and in ultimate $441(18.4 \%)$ cases, appendectomy was completed at the side of the opposite techniques consisting of 
hysterectomy, and colectomy. The prevalence of appendicitis and appendectomy have been better in ladies. The survey revealed, higher incidence price amongst 16-30 yr age organization accompanied via $31-45$ and $1-15$ years.

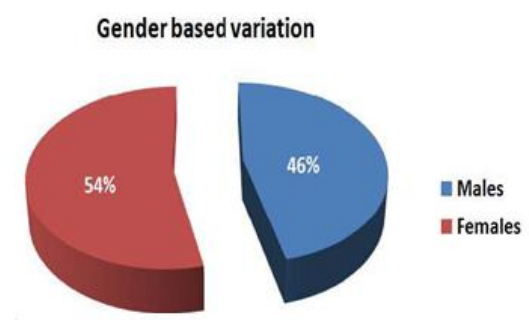

Fig1. Shown the gender based variation in the frequency of appendectomy cases.

\section{APPENDICITIS AND ITS TYPES}

Appendicitis is irritation of the appendix. Appendicitis commonly provides with right iliac fossa stomach pain, nausea, vomiting, and reduced appetite. But, one 0.33 to a half of people do not have these standard symptoms and signs and symptoms. Intense headaches of a ruptured appendix consist of huge unfold, the painful infection of the internal lining of the stomach wall and sepsis.

Appendicitis is due to a blockage of the hollow part of the appendix, maximum normally through a calcified "stone" fabricated from feces. However inflamed lymphoid tissue from a viral infection, parasites, gallstone or tumors may also purpose the blockage. This blockage results in improved pressures inside the appendix reduced blood drift to the tissues of the appendix, and bacterial increase inside the appendix causing irritation. The mixture of infection reduced blood float to the appendix and distention of the appendix reasons tissue harm and tissue death.[3] If this technique is left untreated, the appendix might also burst, liberate bacteria into the belly cavity, main to intense belly ache and improved headaches. There are four exclusive training like Acute appendicitis, Sub-acute appendicitis, Recurrent appendicitis

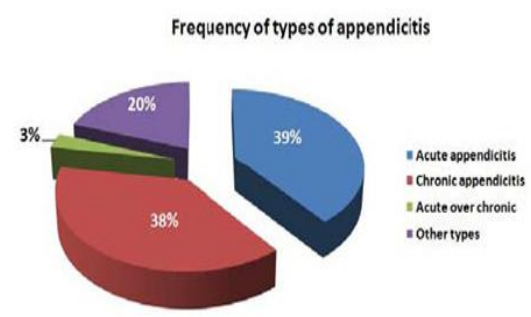

Fig2. Types of appendicitis

\section{Acute Appendicitis}

Acute appendicitis is the most common surgical condition of the abdomen. Acute appendicitis may occur at all ages but is most commonly seen in the second and third decades of life. Acute appendicitis is a rapidly progressing inflammation of a small part of the large intestine called the appendix. Acute appendicitis is a medical emergency that generally requires prompt removal of the appendix to prevent life-threatening complications, such as ruptured appendix and peritonitis. In contrast, chronic appendicitis develops slowly, has milder symptoms, and can often be treated with antibiotics. Chronic appendicitis is far less common than acute appendicitis.

\section{Sub-acute Appendicitis}

The collection given in acute appendicitis is not inevitable. Some episodes of acute appendicitis seemingly subside spontaneously before they attain the extreme level. This is known as-as sub-acute appendicitis.

\section{Recurrent Appendicitis}

If a full-blown appendicitis does not take place, the appendix might also develop into a 'grumbler' precipitating recurrent attacks. That is known as recurrent appendicitis. Those attacks are typically milder. The patients remain symptom-unfastened among attacks and the bodily exam is normal. Barium enema $\mathrm{X}$-ray regularly shows ordinary filling of the appendix due to the disappearance of obstruction.

\section{Chronic Appendicitis}

Occasionally pathological exam of the appendix might also reveal thickening and scarring suggesting vintage, healed acute inflammation. That is called as persistent appendicitis. Patients with such appendicitis regularly complain of the continual proper lower belly ache. The resected appendix indicates fibrosis of the appendicular wall, evidence of antique mucosal ulceration and scarring and infiltration by continual inflammatory cells to be specified as continual appendicitis.

\section{ENSEMBLE LEARNING}

The ensemble techniques are mastering algorithms that create a set of classifiers and then classify new information points through taking a weighted vote of their predictions/classification. Ensemble mastering is a system getting to know version wherein multiple rookies are trained to solve the identical hassle. In distinction to regular gadget gaining knowledge of techniques which attempt to research one speculation from the education dataset, ensemble strategies try to construct a hard and fast of hypotheses and combine them to use for prediction.

An ensemble includes a number of learners which are normally called as base freshmen. The generalization capability of an ensemble is generally a great deal more potent than base inexperienced persons. In point of fact, ensemble mastering is quite exciting due to the fact that it has a successful to boost vulnerable inexperienced persons which are relatively higher than random estimate to strong learners that can make very correct predictions. So, the base learners also are referred as vulnerable novices. Normally, an ensemble is created in two steps. step one, some of base newcomers is produced sequentially. The second one step, base newcomers are blended to apply, where some of the most famous strategies are majority voting for class and weighted averaging for regression techniques.

Normally, to get a good ensemble, the base newcomers ought to be as greater accurate as possible, and as extra assorted as feasible. This has been formally proven [5], and accentuated by many other populaces. There are numerous powerful processes for computing the accuracy of beginners, such as move-validation, preserve-out check, etc. there are many a success ensemble strategies inclusive of Boosting [6],[7], Bagging [8] and Stacking [9]. on this work, the AdaBoost algorithm is used in category.

\section{AdaBoost set of rules}

Boosting approach [10] is an ensemble class such that each classifier has a weight that is derived from the correctness of studying. The learned fashions are used to expect the unknown records by way of most people vote. The most famous method is AdaBoost ensemble mastering.

The AdaBoost algorithm [11] is a well-known approach to construct ensembles of classifiers with very superb overall 
performance. This set of rules takes education facts and defines vulnerable classifier capabilities for each pattern of the education dataset. Classifier function takes the sample as a controversy and produces fee zero or 1 within the case of a binary category undertaking and weight element for every classifier. The pseudo-code of AdaBoost is shown in underneath:

AdaBoost Algorithm:

Input: Sample Dataset D

Base Learning Algorithm L;

Number of Training Rounds $T$.

Process:

Initialize the weight distribution $\left(D_{t}\right)$

For each learning rounds

1.Train base learner using distribution weight

2. Measure base learner error

3. Find the weight of base learners

4. Update the distribution, until the distribution factor reached $(\mathrm{Dt}+1)$.

End

Output: T Classifiers

\section{EXPERIMENTS AND RESULTS}

Simulation experiments in this research are finished on a laptop with Intel corei3 @ $2.00 \mathrm{GHZ}$ CPU and 4GB reminiscence. The technique is designed on a platform of Matlab 2014a at the operation machine windows 8.1. The datasets used in this research had been prepared from actual samples for tomato at exceptional diseases, which were accrued from a neighborhood market. The dataset consists of 500 pictures of five specific appendicitis and every a hundred snap shots respectively. The records are accrued manually from nearby hospitals. There are 5 different instructions like Acute appendicitis, Sub-acute appendicitis, Recurrent appendicitis, chronic appendicitis and regular. Ensemble learning algorithm is used to classify the one-of-a-kind diseases appropriately based totally on the capabilities; it has been trained and examined.

In the preceding work, MRI picture is taken and numerous filtering strategies are applied like LEE and FROST methods are used for noise elimination and Marker-managed Watershed method is used for segmentation of appendicitis. Location of hobby (ROI) approach is used to extract the correct part of appendix photograph. The feel features of the segmented ROI are Gary level Co-prevalence Matrix (GLCM) Autocorrelation, contrast, Correlation, Dissimilarity, strength, Entropy, and Homogeneity. Shape capabilities extracted thru the regionprob set of rules like Eccentricity, Orientation, Solidity, and Perimeter.

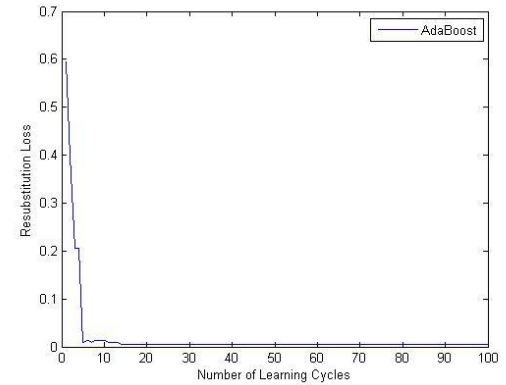

a) Resubstitution error

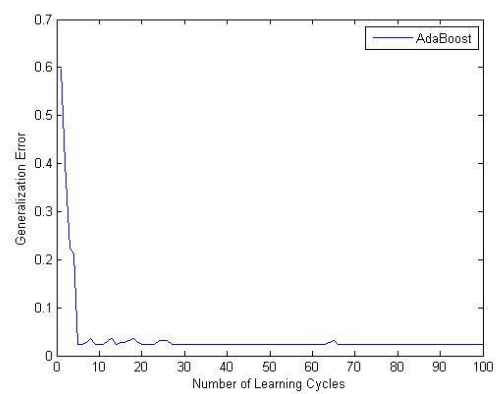

b) Generalization error

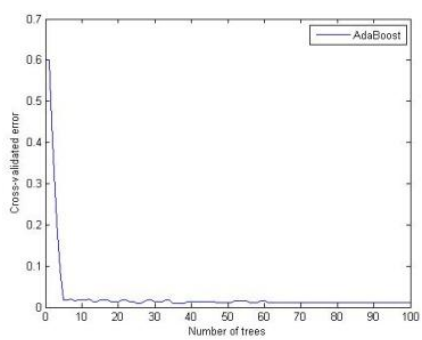

c) Cross-validation error

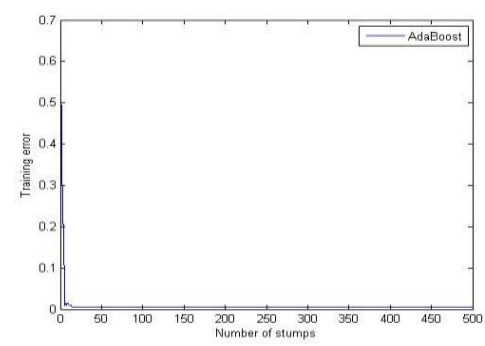

d) Training error

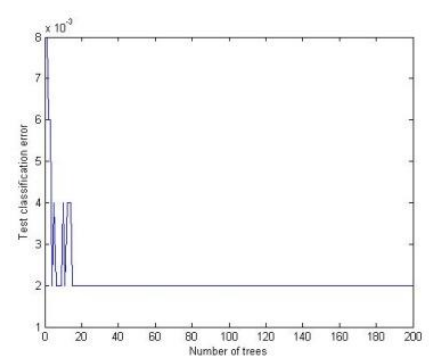

e) Classification error 


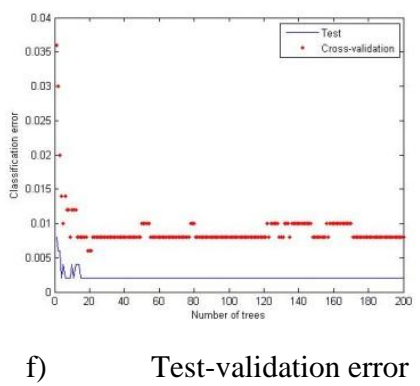

Fig3. Evolution Measures

The above parent (Fig.3) indicates the mistake rate of Resubstitution Loss mistakes, Generalization mistakes, goValidation mistakes, and training blunders. The AdaBoost set of rules plays nicely and reaches maximum new release with low blunders price. The error fees are computed for training dataset.

The multi-elegance ensemble techniques like AdaBoost are used to evaluate the accuracy. The AdaBoost algorithms out play well in predicting tomato diseases with the excessive accuracy charge of approximately $99 \%$ when weigh towards to other getting to know strategies. The next desk (Table1) has proven the accuracy of numerous strategies and its accuracy rate, ensemble size, and accuracy loss rate. The underneath image (Fig4) demonstrates the accuracy output and error evolution measures.

\section{Table1. Evolution measures}

\begin{tabular}{|l|l|l|l|}
\hline $\begin{array}{l}\text { Algorithm } \\
\text { / Accuracy }\end{array}$ & Accuracy & $\begin{array}{l}\text { Ensemble } \\
\text { Size }\end{array}$ & $\begin{array}{l}\text { Accuracy } \\
\text { Loss }\end{array}$ \\
\hline AdaBoost & $99.20 \%$ & 1626526 & 0.0233 \\
\hline & \\
\hline & \\
\hline & \\
\hline
\end{tabular}

Fig4. Ensemble learning accuracy

\section{CONCLUSION}

In the preceding work, MRI photo is preprocessed and segmented. From the segmented photograph, specific sorts of features are extracted particularly grey-level Co-prevalence Matrix (GLCM) and shape capabilities. Ultimately, ensemble AdaBoost studying method is used to categorise appendicitis. The Experimental end result indicates that Adaboost method outperforms nicely in prediction of appendicitis greater appropriately with above $99 \%$ of classification accuracy. Regards to destiny enhancement of this painting focused on increasing dataset and this try highlights at the lee and frost methods for filtering of appendix picture. As for the enhancement of appendicitis prognosis algorithm, other filtering techniques can be tested and as compared for selecting the extra algorithm.

\section{REFERENCES}

[1] M. C. Horattas, D. P. Guyton, and D. Wu "A reappraisal of appendicitis in elderly," Am. J Surgery, 1990; vol. 160, pp. 291-293, 1990.

[2] H. C. Yeh and J. G. Rabinowitz, "Ultasonography of gastro intestinal tract," Semin Ultrasound CT MR, vol. 3, no. $331,1982$.

[3] H. V. Nghiem and R. B. Jeffrey Jr., "Acute appendicitis confined to the appendiceal tip: evaluation with graded compression sonography," J. Ultrasound Med., vol. 11, no. 5, pp. 205-207, 1992.

[4] R. B. Jeffrey, Jr., F. C. Laing, and R . R. Townsend, "Acute appendicitis: sonographic criteria based on 250 cases," Radiology, vol. 167, no. 2, pp. 327-329, 1988.

[5]. Krogh, A., Vedelsby, J.: Neural network ensembles, cross validation, and active learning. In Tesauro, G. Touretzky, D.S., Leen, T.K., eds.: Advances in Neural Information Processing Systems 7. MIT Press, Cambridge, MA (1995) 231-238

[6]. Schapire, R.E.: The strength of weak learnability. Machine Learning 5(2) (1990) 197-227.

[7]. Freund, Y., Schapire, R.E.: A decision-theoretic generalization of on-line learning and an application to Boosting. Journal of Computer and System Sciences 55(1) (1997) 119-139.

[8]. Breiman, L.: Bagging predictors. Machine Learning 24(2) (1996) 123-140

[9]. Wolpert, D.H.: Stacked generalization. Neural Networks 5(2) (1992) 241-260

[10] Hastie T, Tibshirani R, Friedman J. The elements of statistical learning, Data Mining, Inference, and Prediction. Stanford, California.2nd ed. Springer. 2001.

[11] Yoav Freund, and Robert E. Schapire, "Experiments with a new boosting algorithm", Proceedings $13^{\text {th }}$ International Conference on Machine Learning, Vol. 96, pp. 148-156, 1996 